\title{
Strong lens search in the ESO public Survey KiDS
}

\author{
N. R. Napolitano, G. Covone, N. Roy, C. Tortora, F. La Barbera, M. Radovich, \\ F. Getman, M. Capaccioli, A. Colonna, M. Paolillo, G. A. Verdoes Kleijn, \\ L.V.E. Koopmans and KiDS collaboration
}

\begin{abstract}
We have started a systematic search of strong lens candidates in the ESO public survey KiDS based on the visual inspection of massive galaxies in the redshift range $0.1<z<0.5$. As a pilot program we have inspected 100 sq. deg., which overlap with SDSS and where there are known lenses to use as a control sample. Taking advantage of the superb image quality of VST/OmegaCAM, the colour information and accurate model subtracted images, we have found 18 new lens candidates, for which spectroscopic confirmation will be needed to confirm their lensing nature and study the mass profile of the lensing galaxies.
\end{abstract}

\section{Dark matter in galaxy cores}

The dominant role of cold dark matter (CDM) in shaping structures in the Universe is now very well constrained and understood on the largest scales, but less on the scales of individual galaxy haloes. The assembly and evolution of CDM haloes can be studied in detail with $\mathrm{N}$-body simulations, which predict that the CDM density profile, $\rho_{\mathrm{CDM}}(r)$, are well described by the so-called NFW profile with $\rho_{\mathrm{CDM}}(r) \propto$ $r^{-3}$ in the outer regions, and $\rho_{\mathrm{CDM}}(r) \propto r^{-\alpha}$, in the centre (with $\alpha=-1,[15]$; $\alpha=-1.5[14]$ ). However, N-body simulations only follow the evolution of CDM

N.R. Napolitano e-mail: napolita@na.astro.it C. Tortora, F. La Barbera, F. Getman INAF - Osservatorio Astronomico di Capodimonte, Salita Moiariello, 16, 80131 - Napoli, Italy

G. Covone, N. Roy, M. Capaccioli, A. Colonna, M. Paolillo

Dipartimento di Scienze Fisiche, Università di Napoli Federico II, Compl. Univ. Monte S. Angelo, 80126 - Napoli, Italy

M. Radovich

INAF - Osservatorio Astronomico di Padova-vicolo Osservatorio 5 - 35122 Padova,Italy

Gijs A. Verdoes Kleijn, L.V.E. Koopmans Kapteyn Astronomical Institute, University of Groningen, P.O. Box 800, 9700 AV Groningen, the Netherlands 
particles, not including the complex physics of gas and stars, which can alter the CDM profiles (e.g., via adiabatic contraction, [10] ).

The study of the CDM profile in the inner regions of ETGs has received much interest, due to the large amount of available data samples (e.g., [11]; [19]). However, it is made difficult by the degeneracy between the shape of the CDM profile and that of the stellar Initial Mass Function (IMF; [7]; [20].)

\section{Strong Lensing to study the mass density of galaxies}

Strong lensing (SL hereafter) is an ideal tool to perform accurate studies of the CDM distribution of intermediate and massive galaxies (e.g. with velocity dispersions, $\sigma_{v}>180 \mathrm{~km} \mathrm{~s}^{-1}$ ) independently of the dynamical status of the mass within the arc features. SL studies, often in combination with stellar dynamics in the galaxy central regions, have found that the light and halo profiles conspire to have a total mass density profile which is nearly isothermal ([12]; [3]; [2]; [5]; [16]), i.e. a total-mass density slope of $\alpha \sim-2$. This "conspiracy", once thought to be universal, has been recently found not to be so, as lower mass galaxies might have steeper $\alpha$ ([8]; [21]). The physical reasons for this systematic variation of the observed slope resides in the way baryons and CDM are mixed, after having been assembled over time [17]. Such detailed studies have been performed over samples of nearby early-type galaxies and a similar systematic study is ongoing at higher-z SL systems (e.g., by SLACS, [4]; SL2S, [18]), where SL is much more effective than standard dynamical studies. For this reason it is becoming of fundamental importance to enlarge the sample of SL systems either in the galaxy parameter space (e.g. toward less massive systems, like sub- $L_{*}$ ) and in redshift space.

\section{Why galaxy-galaxy SL in the ESO Public Survey KiDS?}

The ESO public survey KiDS (Kilo Degree Survey, [6]) is a multi-band (ugri) imaging survey, designed to take advantage of the superb seeing of Paranal and high image quality of the VST/OmegaCAM. Its primary usage is weak lensing measurements over 1500 sq. deg of the sky.

As such, KiDS is particularly suitable for a systematic census of lensing galaxies based on the identification of arc-like structure from intermediate to massive galaxies (i.e., with velocity dispersion of the order of $200 \mathrm{~km} \mathrm{~s}^{-1}$ or larger), to galaxy groups and galaxy clusters, thus covering a wide range of lens masses. Indeed, the Einstein ring writes easily as a function of the velocity dispersion as $R_{\mathrm{E}} \sim 1^{\prime \prime} \times\left(\sigma_{v} / 220 \mathrm{~km} \mathrm{~s}^{-1}\right)^{2}$, and for a typical $F W H M \sim 0.7^{\prime \prime}$ in $r$-band, we can expect to observe lensing arcs of gravitational structures of $\sigma_{v}>180 \mathrm{~km} \mathrm{~s}^{-1}$.

The search for SL in KiDS is expected to provide up to thousands galaxy rings (e.g. by extrapolating CFHT-LS numbers, [16]). This can be performed only with 

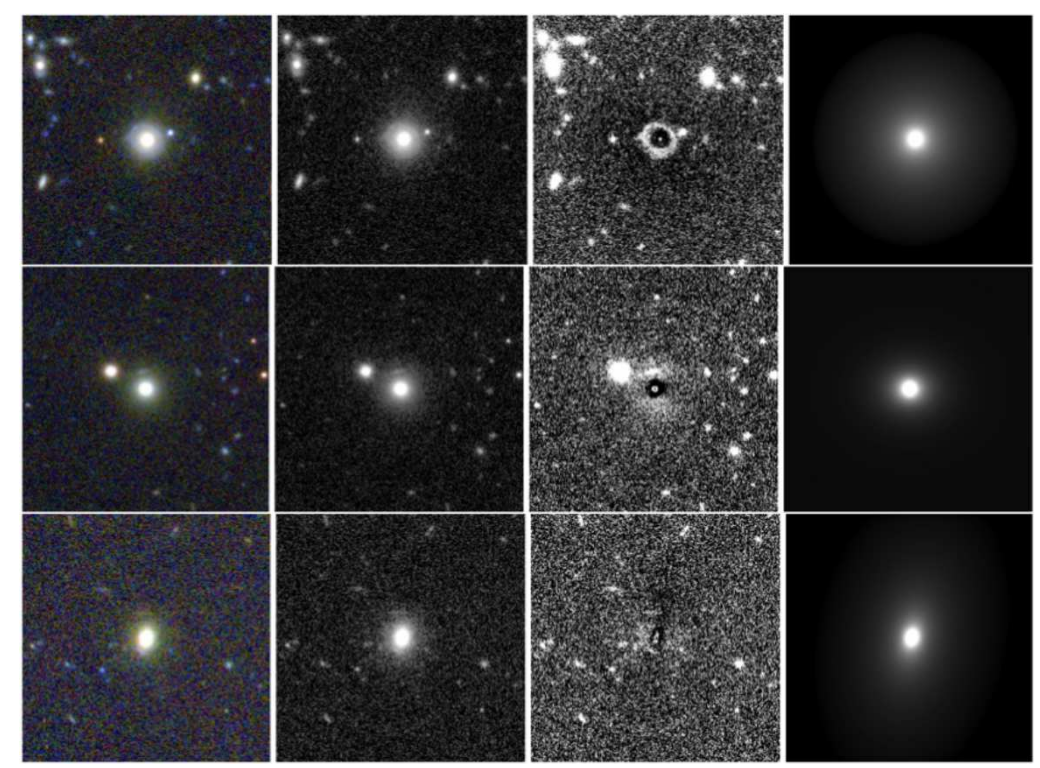

Fig. 1 Results from the visual inspection of SL candidates. Top rows show two high-confidence candidates, bottom is a less secure one. At each row we show: 1) RGB colour image, 2) $r$-band image; 3) model subtracted image; 4) 2DPHOT galaxy model. In the top row, an Einstein ring is evident and shows up clearly in the residual image. In the middle row a blue arc is also evident in the colour image and nicely spotted into the residual image. In the bottom row a faint blue arc is seen in the colour image in the bottom edge of the redder central galaxy, which shows up slightly clearer in the model subtracted image. Estimated $R_{E}$ are $6-8$ arcsec.

(semi-)automated techniques ([1]; [9]), as potential candidates in KiDS can be of the order of thousands per square degree, making visual inspection inapplicable. However, lensing finding techniques still suffer from a number of issues yet to be solved, affecting the completeness and reliability of their catalogs and need to be trained over visually-inspected samples.

\section{Selection of SL candidates via visual inspection}

Due to the yet limited extension of the area (150 sq. deg.) included in the KiDS ESOData Release 2 (ESO-DR2), we have decided to start a visual search based on simple criteria: 1) sources with SDSS spectroscopic redshifts between $z=0.1$ and $z=0.5$ and 2) sources brighter than $r=20$, which together maximise the SL probability for the depth allowed by KiDS images. These criteria were restrictive and biased toward larger $\mathrm{R}_{E}$ easier to spot by visual inspection. In order to quantify the performance of our search, we decided to start on some control fields, overlapping with the SDSS, and selected $\sim 600$ lens candidates in $\sim 100$ sq.deg, including a ten of SLACS lenses. 
The visual inspection (performed by six observers) was based on a) the galaxy $r$-band image ( $r$-band is chosen as reference since it is the deepest and highest quality among KiDS wavebands), b) a three-colour combined image to find a gradient in colour between the candidate lens galaxy and the arc, c) the 2DPHOT model [13] subtracted image to detect residual arc features. Examples of lens candidates are in Figure 1). Following this procedure, we ended up with a list of 18 new potential lens candidates, half of which have high significance.

The spectroscopic follow-up will provide us with the confirmation of the lensing nature of the lens candidates and the independent kinematic information to constrain the mass profile of the lensing galaxies.

\section{References}

1. Alard, C. 2007, MNRAS, 382, L58

2. Auger, M. W., Treu, T., Bolton, A. S., et al., 2010, ApJ, 724, 511

3. Bolton, A. S., Burles, S., Koopmans, L. V. E., Treu, T., Moustakas, L. A., 2006, ApJ, 638, 703

4. Bolton, A. S., Burles, S., Koopmans, L. V. E., et al. 2008, ApJ, 682, 964

5. Chae, K. H., Bernardi, M., Kravtsov, A. V., 2014, MNRAS, 437, 3670

6. de Jong, J. T. A., Kuijken, K., Applegate, D., et al., 2013, The Messenger, 154, 44

7. Dutton, A. A., Mendel, J. T., Simard, L., 2012, MNRAS, 422, L33

8. Dutton, A. A., \& Treu, T., 2014, MNRAS, 438, 3594

9. Gavazzi, R., Marshall, P. J., Treu, T., Sonnenfeld, A., 2014, ApJ, 785, 144

10. Gnedin, O. Y., Kravtsov, A. V., Klypin, A. A., Nagai, D., 2004, ApJ, 616, 16

11. Hyde, J. B., \& Bernardi, M., 2009, MNRAS, 396, 1171

12. Koopmans, L. V. E., Treu, T., Bolton, A. S., Burles, S., Moustakas, L. A., 2006, ApJ, 649, 599

13. La Barbera, F., de Carvalho, R. R., Kohl-Moreira, J. L. et al., 2008, PASP, 120, 681

14. Moore, B., Governato, F., Quinn, T., Stadel, J., Lake, G., 1998, ApJ, 499, L5

15. Navarro, J. F, Frenk, C. S., White, S. D. M., 1996, ApJ, 462, 563

16. Oguri, M., Rusu, C. E., Falco, E. E., 2014, MNRAS, 439, 2494

17. Remus, R. S., Burkert, A., Dolag, K., et al. 2013, ApJ, 766, 71

18. Ruff, A. J., Gavazzi, R., Marshall, P. J., Treu, T., Auger,M. W., Brault, F., 2011, ApJ, 727, 96

19. Tortora, C., La Barbera, F., Napolitano, N. R., de Carvalho, R. R., Romanowsky, A. J., 2012, MNRAS, 425, 577

20. Tortora, C., Romanowsky, A. J., Napolitano, N. R., 2013, ApJ, 765, 8

21. Tortora, C., La Barbera, F., Napolitano, N. R., Romanowsky, A. J., Ferreras, I., de Carvalho, R. R., 2014, MNRAS, 445, 115 